\title{
特別講演
}

\section{Electron Microscopic Autoradiographic Study of Lipoprotein Entry into Arterial Wall}

\author{
Paris Constantinides* \\ University of British Columbia Medical School, Pathology Department, \\ Vancouver, Canada
}

A basic assumption behind most atherosclerosis research of the last twenty years has been the idea that the giant lipoprotein molecules which circulate in our blood percolate all the time through the walls of our arteries (moving from the lumen through the intima and media into the adventitia) and that when there are too many of them, they get into the wall faster than they get out of it, and, therefore, begin to accumulate there.

In recent years, however, our own studies and those of others have led us to suspect that far from being freely permeable, the normal arterial endothelium has a very low permeability for large lipoprotein molecules.

We therefore proposed a very different hypothesis at the Tokyo, Frankfurt and Helsinki Symposia of 1969 and at the Lindau Symposium of 1970, as a basis for future experimental work:

We postulated that under normal conditions mostly small molecules cross into the arterial wall to take care of its metabolic needs. Most of the big molecules, such as the lipoproteins, would leave the vascular

* Professor of Pathology Department British Columbia University, Canada Visiting Professor of Tokyo Ika-Shika National University tree at its finest terminal twigs, namely the capillaries and venules, in order to get to the various organs where they are urgently needed to supply energy and building blocks for new cell membranes. And if we stop to think about it for a moment, arteries really represent canals for long distance transport. It would not make any biological sense if nature allowed these valuable large molecules to be lost in the walls of the canals before they reach their destination.

When, however, an artery is injured in any of a dozen ways, then injury changes the endothelium, it "opens doors" in it and allows large quantities of lipoprotein molecules to penetrate into the arterial wall where they accumulate because they cannot get out and they cannot be handled easily; they are not expected there.

What insults could open doors in the arterial endothelium? We thought that most of the agents that promote the deposition of lipids into arteries should be able to do this, namely hypertension, immune insults, diabetes, nicotine, and - above all - prolonged hyperlipemia.

Some recent studies have given partial support to this concept, notably those of Dr. Adams, Dr. Gore and Drs. Scott and Hurley. These workers injected intraven- 
動脈硬化 Vol.1

ously labelled cholesterol or lipoprotein labelled in its protein component and, using purely quantitative measurements of tissue radioactivity, found an increased entry of both these materials into the atherosclerotic, as compared to the normal arterial wall of rabbits, and a much greater entry of lipoprotein into the liver than the aorta of human patients.

We therefore thought that it would be very interesting to test this hypothesis further by visualising directly, through electron microscopic autoradiography, the transendothelial movements of lipoprotein molecules labelled in their lipid component and manufactured by the animal itself in a physiological manner, from radioactive cholesterol given by mouth. Such a type of study had not yet been accomplished successfully, to our knowledge, for 2 reasons: First, the labelled cholesterol was extracted out of the tissues by the lipid solvents used during processing for electron microscopy, and secondly, any label given by mouth was diluted enormously over the vast inner surface of the entire vascular system.

We overcame the first difficulty by fixing the tissues in the presence of digitonin, a compound that forms an insoluble precipitate with free cholesterol and has been used recently by Drs. Ökrös, Scallen and Dietert, Napolitano and others to demonstrate this steroid ultrastructurally in liver, adrenal and myelin. The second difficulty was overcome by giving rabbits the extremely "hot" dose of $15 \mathrm{mCi} / \mathrm{kg}{ }^{3} \mathrm{H}$-Cholesterol by stomach tube, spread over 5 days.

We fed the labelled cholesterol to several normal, chronically hyperlipemic, DOCAtreated, and serum-sick rabbits, killed these animals 6 hours after their last ${ }^{3} \mathrm{H}$ -
No. 3 October 1973

steroid feeding and examined thick, unstained sections from their arteries, liver and heart under an electron microscope, after coating them with photographic Ilford L 4 emulsion, exposing them for 6 months and developing them.

The results can be summarized as follows:

We found that the arterial endothelium of normal animals had an extremely low permeability for the labelled lipd circulating in the blood after ${ }^{3} \mathrm{H}$-Cholesterol feeding, while the capillary endothelium in the liver and the heart was highly permeable to it.

By contrast, the arterial endothelium of hyperlipemic, DOCA-treated and serumsick animals proved extremely permeable to the labelled lipid, allowing it to cross freely into the subendothelial space. In the case of the hyperlipemic animals, most of the lipid moved through the cytoplasm of the endothelial cells, but some of it also entered - free or within immigrant monocytes - through opened endothelial junctions that we observed under these conditions.

Future research will show in what form and through what mechanism the lipid crosses the endothelial cytoplasm, but it seems already certain that the lipid which enters through the opened junctions enters as a whole lipoprotein molecule.

In conclusion, our results provide direct visual evidence for the hypothesis I outlined at the beginning, that the normal arterial endothelium has an extremely low permeability for physiologically introduced large lipoprotein molecules, but that certain pathological conditions such as prolonged hyperlipemia, hypertension and immune insults change it and make it very permeable to them. 